
estresse e humor de corredores? Cuadernos de Psicología del Deporte, Vol 19(3), 254-261

\title{
A prática de Hatha Yóga melhora os sintomas de estresse e humor de corredores?
}

\section{Does Hatha Yóga single session can improves the stress and mood symptoms of runners?}

\section{La práctica de Hatha Yóga mejora los síntomas de estrés y humor de corredores?}

Roberta L. Rica ${ }^{1}$, Adriano Fortes Maia ${ }^{2}$, Gerson dos Santos Leite ${ }^{3}$, João Marcelo de Queiroz Miranda ${ }^{4}$, Alana de Sena Souza, Welmo Alcântara Barbosa ${ }^{2}$, Danilo Sales Bocalini ${ }^{2}$

${ }^{1}$ Departamento de Educação Física. Universidade Estacio de Sá, Vitoria, ES, Brasil. ${ }^{1}$ Laboratorio de Físiologia e Bioquimica Experimental. Centro de Educação Física e Desporto da Universidade Federal do Espirito Santo, Vitoria, ES, Brasil. ${ }^{3}$ Departamento de Educação Física. Universidade Nove de Julho,

São Paulo, SP, Brasil. ${ }^{3}$ Departamento de Educação Física. Universidade Cidade de São Paulo, São

Paulo, SP, Brasil.

\section{Resumo}

Introdução: A prática de Yóga é reconhecida por seus diversos benefícios, contudo considerando a prática como estratégia de gerenciamento do estresse gerados pelas cargas de treino em atletas não está claro na literatura. Objetivo: avaliar os efeitos de uma sessão de prática de Hatha Yóga (HY) nos sintomas de stress e no estado de humor de corredores. Metodologia: Após uma semana de altas cargas de treinamento (volume de 45,0 $\pm 15,0 \mathrm{~km}$ percorridos na semana) vinte corredores amadores com idade $\geq 18$ anos participaram voluntariamente de uma sessão de HY sendo composta de 10 minutos de exercícios de controle da respiração, 15 minutos de exercícios de aquecimento, 50 minutos de posturas de yoga e 10 minutos de relaxamento. Antes (An) e apos (Ap) a prática todos os corredores responderam o inventário de sintomas de stress e questionário de humor (POMS). Resultado: $67 \%$ dos indivíduos foram classificados em fase de resistência e 33\% em fase de pré-exaustão, após a prática de HY nenhum individuo apresentou sintomas de stress. Em relação aos parâmetros do estado de humor diferenças significativas $(\mathrm{p}<0,01)$ foram encontradas na tensão (An: 12 \pm 2 , Ap: 5 \pm 2 ), depressão (An: 10 \pm 2 , Ap: $2 \pm 2$ ), raiva (An: 13 \pm 2 , Ap: $3 \pm 2$ ), fadiga (An: 15 \pm 2 , Ap: $7 \pm 2$ ), confusão mental (An: 12 \pm 2 , Ap: $5 \pm 2$ ) e vigor (An: 12 \pm 3 , Ap: 20 \pm 3 ) após a pratica de HY. Conclusão: nossos dados indicam que a pratica de HY pode contribuir para minimizar o estresse e as alterações negativas nos estados de humor decorrentes de uma semana intensificada de treinamento.

Palavras chave: corrida, estresse, desempenho, exercício, performance 


\title{
Prática de Hatha Yóga e sintomas de estresse e humor de corredores
}

\begin{abstract}
Problematization: Yóga's practice is recognized for its many benefits, however considering practice as a stress management strategy generated by training loads in athletes is not clear in the literature. Objective: to evaluate the effects of a practice session of Hatha Yóga (HY) on the stress symptoms and mood of runners. Methods: After a week of high training loads (volume of $45.0 \pm 15.0 \mathrm{~km}$ traveled in the week) twenty amateur runners aged $\geq 18$ years participated voluntarily in an HY session consisting of 10 minutes of control exercises breathing, 15 minutes of warm-up exercises, 50 minutes of yoga postures and 10 minutes of relaxation. Before (B) and after (A) practice all runners responded to the inventory of stress symptoms and mood questionnaire (POMS). Results: 67\% of the individuals were classified in the resistance phase and 33\% in the pre-exhaustion phase, after the practice of HY all the individuals did not present symptoms of stress. Regarding the parameters of the mood state, significant differences $(\mathrm{p}<0.01$ ) were found in tension (B: $12 \pm 2, A: 5 \pm 2$ ), depression (B: $10 \pm 2, A: 2 \pm 2),(B: 13 \pm 2, A: 3 \pm 2$ ), fatigue (B: 15 \pm 2 , A: $7 \pm 2$ ), mental confusion (B: $12 \pm 2, A: 5 \pm 2$ ) and vigor (B: $12 \pm 3$, A: $20 \pm 3$ ) after the practice of HY. Conclusion: Our data indicate that the practice of HY can contribute to minimize stress and negative changes in mood states due to an intensified training week.
\end{abstract}

Key words: run, stress, performance, exercise, performance

\section{Resumen}

Introducción: La práctica de Yóga es reconocida por sus diversos beneficios, sin embargo considerando práctica como estrategia de gestión del estrés generados por las cargas de entrenamiento en atletas no está clara en la literatura. Objetivo: evaluar los efectos de una sesión de práctica de Hatha Yóga (HY) en los síntomas de estrés y en el estado de humor de los corredores. Metodología: Después de una semana de altas cargas de entrenamiento (volumen de $45,0 \pm 15,0 \mathrm{~km}$ recorridos en la semana) veinte corredores aficionados con edad $\geq 18$ años participaron voluntariamente de una sesión de HY siendo compuesta de 10 minutos de ejercicios de control de la enfermedad respiración, 15 minutos de ejercicios de calentamiento, 50 minutos de posturas de yoga y 10 minutos de relajación. Antes (A) y después (D) la práctica todos los corredores respondieron el inventario de síntomas de estrés y cuestionario de humor (POMS). Resultado: 67\% de los individuos fueron clasificados en fase de resistencia y 33\% en fase de pre-agotamiento, después de la práctica de HY todos los individuos no presentaron síntomas de estrés. En relación a los parámetros del estado de humor, las diferencias significativas $(\mathrm{p}<0,01)$ se encontraron en la tensión (A: $12 \pm 2$, D: $5 \pm 2$ ), depresión (A: $10 \pm 2$, D: $2 \pm 2$ ), (A: $13 \pm 2$, D: $3 \pm 2$ ), fatiga (A: 15 \pm 2 , D: $7 \pm 2$ ), confusión mental (A: 12 \pm 2 , D: $5 \pm 2$ ) y vigor (A: $12 \pm 3$, D: $20 \pm 3$ ) después de la práctica de HY. Conclusión: nuestros datos indican que la práctica de HY puede contribuir a minimizar el estrés y los cambios negativos en los estados de humor derivados de una semana intensificada de entrenamiento.

Palabras clave: carrera, estrés, rendimiento, ejercicio, rendimiento

\section{INTRODUÇÃO}

Nos últimos anos a corrida de rua teve um crescimento exponencial em todo o mundo e vem ganhando mais adeptos a cada dia, bem como ampliando o número de provas (Dallari 2009; Oliveira 2009; Gotaas 2013; Shipway e Holloway 2016, Salgado e Chacon-Mikahil 2016). Um diferencial para

o crescimento exponencial que a corrida de rua sofreu no Brasil, em especial, se deu pela facilidade do acesso à sua prática, uma vez que ela necessita de uma estrutura física simples, facilmente encontrada em qualquer cidade. Além disso, a modalidade não requer a utilização de muitos materiais, se comparada a outras atividades esportivas (Rojo et al. 2017).

OSociedad
OSeroamericana de
Oeporte




\section{Rica RL, Maia AF, Leite GS, Miranda JMQ, Souza AS, Barbosa WA, Bocalini DS}

Além do crescimento das provas e praticantes, é cada vez mais comum o surgimento de lesões em corredores, por conta da alta carga de treinamento (Knechtli e Nikolaidis 2018). Gomes (2002) sugere que a relação de dose-resposta da carga de treinamento requer a atenção dos especialistas e uma investigação científica contínua e permanente, pois quando aplicada em excesso pode gerar prejuízos aos atletas, como estados de overtraining/estafa (Leite et al. 2012).

O estresse e os estados de humor têm sido utilizados para monitorar atletas e auxiliar nos ajustes das cargas de treinamento, visto que quanto maior for o estresse geral do atleta, maior a chance de condições inadequadas á prática esportiva e a obtenção de melhores resultados esportivos (Ferreira Brandão et al. 2015). Desta forma, treinadores, médicos, nutricionistas e psicólogos do esporte têm procurado diferentes formas de minimizar o estresse e as alterações no estado de humor de atletas.

Estudos (Benvenutti et al. 2017; Shohani et al. 2018) utilizando a prática de Yoga demonstram desfechos favoráveis na atenuando o estresse bem como alterações de humor de indivíduos saudáveis ou com alguma patologia. Benvenutti et al. (2017) demonstraram menor reatividade do cortisol salivar, aumento da autoconfiança apos sessão de Yoga. Shohani et al. (2018) avaliando mulheres encontraram redução da depressão, anxiedade e stress apos 12 sessões de prática de Hatha Yoga.

Sabe-se que o desempenho esportivo é altamente influenciado pelo equilíbrio emocional (Rodrigues et al. 2016; Brandt et al. 2016, Brandt et al. 2017), sendo destacado em atletas de basquetebol (Esfahani et al. 2011; Gomes et al. 2018) e taekwondo (Terry, Lane 2006) relações entre os estados de humor e desempenho esportivo. Desta forma, o interesse por treinadores e pesquisadores por estudos que analisam tanto aspectos fisiológicos, quanto psicológicos de atletas aumentaram consideravelmente. Terry (1995) acrescenta que o monitoramento do estado de humor tem como propósito primário obter indicadores da disposição mental/emocional do atleta, porém é possível identificar também queda de desempenho físico e o estado de overtraining.

Contudo apesar do aumento do interesse nas investigações com o monitoramento das alterações psicológicas e gerenciamento do estresse gerados pelas cargas de treino nos atletas, estudos envolvendo corredores e a pratica de Hatha são escassos. Desta forma, o objetivo deste estudo foi verificar a resposta aguda da prática do Hatha Yóga no estresse e estados de humor de corredores.

\section{MATERIAL E MÉTODOS}

\section{Amostra}

A amostra foi composta por 20 corredores amadores com idade $\geq 18$ anos, com experiência de pelo menos um ano em treinamento de corrida sem comprometimento cognitivo, metabólico ou musculoesquelético. Todos os sujeitos assinaram o Termo de Consentimento Livre e Esclarecido, segundo a Resolução n 466/12 do Conselho Nacional da Saúde, aprovado pelo Comitê de Ética em Pesquisa com Seres Humanos da Universidade Nove de Julho sob o número de protocolo (164.497).

\section{Procedimento}

A sessão de Yoga foi realizada conforme previamente descrito por Tran et al. (2001) e logo após uma semana de altas cargas de treinamento (volume de 45,0 $\pm 15,0 \mathrm{~km}$ percorridos na semana). A sessão consitiu em 10 minutos de exercícios de controle da respiração (Pranayamas), 15 minutos de exercícios de aquecimento, 50 minutos de posturas de yoga (Asanas) e 10 minutos de relaxamento (Savasana). Toda a sessão foi realizada por um instrutor habilitado e experiente.

\section{Parâmetros avaliados}

\section{Composição corporal}

A composição corporal (Tabela 1) foi avaliada conforme publicações anteriores pelo nosso grupo (Serra et al. 2014). A massa corporal (MC) foi determinada em balança digital da marca Toledo 


\section{Prática de Hatha Yóga e sintomas de estresse e humor de corredores}

(modelo 2096PP/2), com capacidade de até $200 \mathrm{~kg}$ e sensibilidade de $50 \mathrm{~g}$. A estatura (ES) foi mensurada em estadiômetro da marca SANNY (modelo ES2030), fixado a parede e calibrado em escala de $2 \mathrm{~mm}$, sendo a medida realizada com o cursor em ângulo de $90^{\circ} \mathrm{em}$ relação à escala, com a voluntária na posição ortostática e pés unidos, procurando posicionar em contato com o instrumento de medida as superfícies posteriores dos calcâneos, região occipital e cinturas pélvica e escapular.

Tabela 1. Parâmetros da composição corporal dos corredores.

\begin{tabular}{|c|c|}
\hline Parâmetros & Media \pm DP \\
\hline Idade (anos) & $45 \pm 0,9$ \\
\hline Estatura $(\mathrm{m})$ & $1,75 \pm 0,9$ \\
\hline Massa corporal $(\mathrm{kg})$ & $84 \pm 1,5$ \\
\hline $\operatorname{IMC}\left(\mathrm{kg} / \mathrm{m}^{2}\right)$ & $27 \pm 5,6$ \\
\hline$\%$ de gordura & $25 \pm 0,8$ \\
\hline Massa gorda $(\mathrm{kg})$ & $21 \pm 0,7$ \\
\hline Massa livre de gordura (kg) & $63 \pm 1,3$ \\
\hline $\mathrm{VO}^{2}(\mathrm{ml} / \mathrm{kg} / \mathrm{min})$ & $39 \pm 24$ \\
\hline
\end{tabular}

Valores expressos em media + desvio padrão (DP). IMC: índice de massa corporal.

Obtidas a MC e a ES foi possível calcular o índice de massa corporal (IMC): $\mathrm{MC} \div \mathrm{ES}^{2}$. A composição corporal foi analisada por espessura de dobras cutâneas, obtidas em sete pontos anatômicos distintos (subescapular, suprailíaca, axilar-média, torácica, tricipital, abdominal, coxa).

\section{Sintomas de estresse}

O Inventário de Sintomas de Stress foi utilizado seguindo previas publicações (Lipp 2005; Camelo e Angerami 2004; Cardoso, Loureiro, NelsonFilho 2004). O instrumento permite um diagnóstico claro da existência de sintomas de estresse, bem como da fase em que se encontra (alerta, resistência, quase exaustão e exaustão) e da sintomatologia predominante, se física ou psicológica. Seguindo às diretrizes do Conselho Federal de Psicologia a analise do instrumento foi realizado por uma profissional habilitada.

\section{Estado de humor}

O estado de humor foi avaliado através da aplicação do questionário Perfil dos Estados de Humor (POMS) conforme previa publicação com corredores (Wittig et al. 1992). Resumidamente o instrumento avalia seis estados subjetivos de humor: tensão $(T)$, depressão $(\mathrm{D})$, raiva $(\mathrm{R})$, vigor $(\mathrm{V})$, fadiga $(\mathrm{F})$ e confusão mental (C). Os fatores $T, D, R, F$ e $C$ são considerados os fatores negativos do humor, sendo o vigor considerado o fator positivo. O distúrbio total de humor (DTH) foi calculado pela soma dos fatores negativos, menos $\mathrm{o}$ fator positivo (DTH = $(\mathrm{T}+\mathrm{D}+\mathrm{R}+\mathrm{F}+\mathrm{C})-\mathrm{V})$, sendo uma medida global do distúrbio de humor. Ao resultado final do DTH, foi somado 100 para que não houvessem resultados negativos. O perfil de humor com alto valor de vigor e baixos valores para as outras variáveis é denominado "perfil iceberg", sendo representativo de uma saúde mental positiva. Foi orientado aos participantes responderem de acordo como eles estavam se sentindo "agora, neste exato momento".

\section{Estatística}

O teste de D'Agostino-Pearson foi aplicado para análise da distribuição e normalidade. Para efeito das comparações foram utilizados os testes " $\mathrm{t}$ " de Student ou $\mathrm{X}^{2}$ conforme necessário. Os dados são apresentados sob a forma de médias \pm erro padrão da média. As analises foram realizadas com auxílio dos programas Prism 4.0 (GraphPad Softwares Inc., San Diego, CA, USA) com nível de significância estabelecido para as análises de $\mathrm{p}<0,05$.

\section{RESULTADOS}

Conforme ilustrado na figura 1 , antes da sessão de HY 13 (67\%) indivíduos foram classificados em fase de resistência e 7 (33\%) em fase de préexaustão. Após a prática de HY todos os indivíduos não apresentaram sintomas de stress. 


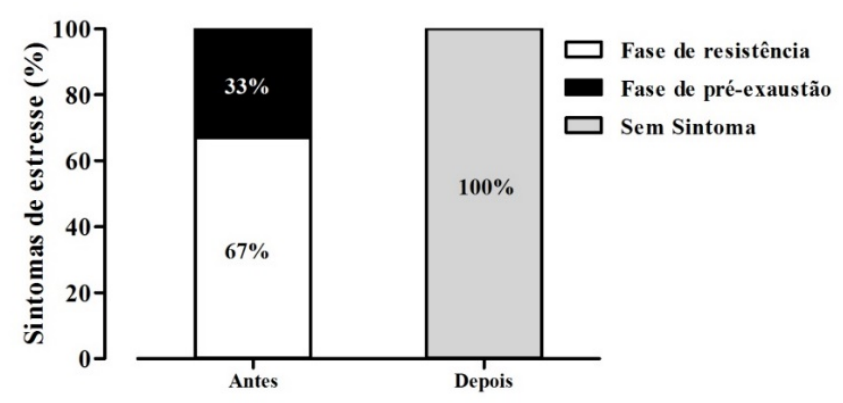

Figura 1. Distribuição percentual (\%) dos parâmetros de sintomas de estresse dos corredores antes e após a sessão de Hatha Yóga.

Considerando os parâmetros de humor (Figura $2)$ diferenças significativas $(\mathrm{p}<0,01)$ foram encontradas em todos os parâmetros após a pratica de HY tensão (An: $12 \pm 2$, Ap: $5 \pm 2$ ), depressão (An: 10 \pm 2 , Ap: $2 \pm 2$ ), raiva (An: $13 \pm 2$, Ap: $3 \pm 2$ ), fadiga (An: $15 \pm 2$, Ap: $7 \pm 2$ ), confusão mental (An: $12 \pm 2$, Ap: $5 \pm 2$ ) e vigor (An: $12 \pm 3$, Ap: $20 \pm 3$ ).

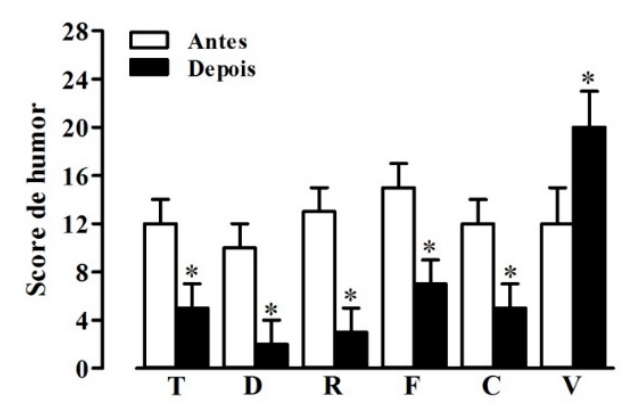

Figura 2. Valores médios \pm desvio padrão dos scores de humor dos corredores antes e depois da da sessão de Hatha Yóga para os parâmetros de tensão (T), depressão (D), raiva (R), vigor (V), fadiga (F) e confusão mental $(\mathrm{C}) .{ }^{*} \mathrm{p}<0,01$ para teste "t "de student pareado.

Considerando o distúrbio de humor redução significativa (Antes: $49 \pm 4$, Depois: $4,5 \pm 2 ; \mathrm{p}<0,001$ ) foi encontrada após a prática de HY.

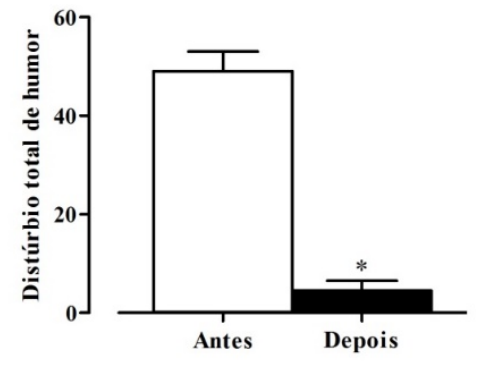

Figura 3. Valores médios \pm desvio padrão dos scores do distúrbio total de humor antes e depois da pratica de uma sessão de Hatha Yóga.* $p<0,01$ para teste " $t$ "de student pareado.

\section{DISCUSSÃ̃}

O principal objetivo deste estudo foi verificar se haveria benefício na prática de Hatha Yóga por corredores amadores após uma semana de treinamentos intensos. O HY foi capaz de minimizar o estresse e as alterações de humor ocorridas devido a grandes cargas de treinamento.

$\mathrm{O}$ estímulo vindo do treinamento induz a adaptação ao treino e é relativo ao estresse fisiológico imposto ao atleta (Impellizzeri et al. 2004). A variação desta carga (estresse fisiológico) aplicada na semana é o que parece contribuir para o aumento do desempenho esportivo (Fry, Morton e Keast, 1992b; Foster et al., 1995), principalmente quando se alternam treinos intensos com treinos leves (Fry, Morton e Keast, 1992a). Ou seja, o treinamento dos atletas aumentará os níveis de estresse, podendo levalos a fase de exaustão quando não foram bem gerenciados (Leite et al 1992). O Inventário de Sintomas de Stress utilizado neste estudo permite um diagnóstico que avalia se a pessoa tem estresse e em qual fase se encontra (Camelo e Angerami 2004). Antes da aula de HY os atletas encontravam-se em padrões intermediários de estresse, nas fases de resistência e de pré-exaustão, o que foi minimizado pela prática do HY, deixando os atletas sem sintomas de estresse. Tal achado pode ser interessante, pois a prática do HY poderia ser implementada na rotina de 


\section{Prática de Hatha Yóga e sintomas de estresse e humor de corredores}

atletas buscando minimizar o estresse causado pelo treinamento e a rotina diária dos atletas.

Outro achado interessante é que as alterações nos estados de humor também foram amenizadas, de forma significativa, pelo HY. A avaliação do estado emocional de atletas durante treinamentos $\mathrm{e}$ competições tem recebido considerável atenção de pesquisadores do mundo todo (Vieira et al., 2010; Kentta, Hassmen, Raglin, 2006), pois um estado emocional adequado é apontado como um dos fatores decisivos ao rendimento esportivo e, para alguns autores, pode explicar desempenhos exitosos ou não (Calmeiro e Tenenbaum 2007; Rohlfs et al. 2004). Um maior volume nos treinamentos promove alterações nos estados emocionais, principalmente aumentando os estados emocionais negativos e diminuição do estado emocional positivo (Vigor) e, quando o volume diminui há melhora nos estados emocionais (Brandt et al. 2010; Caulfield, Karageorghis, 2008; Pierce Jr. 2002; Suay, Ricarte, Salvador, 1998; Morgan et al. 1987; Morgan et al.1988). Nota-se, que o distúrbio total de humor também teve uma redução significativa no presente trabalho, corroborando com a diminuição do estresse dos atletas após a prática do Hatha Yóga e com o achado de outras pesquisas (Wittig et al. 1992; Saw et al. 2016).

Desta forma, parece interessante que outros estudos verificarem a inclusão do Hatha Yóga na rotina semanal dos atletas e entender a resposta crônica aos estímulos de treinamento, buscando assim, minimizar o estresse geral e as alterações nos estados de humor dos atletas devido a carga de treinamento.

\section{CONCLUSÃO}

Uma sessão de Hatha Yóga logo após uma semana de altas cargas de treinamento parece contribuir para minimizar o estresse e as alterações negativas nos estados de humor decorrentes de uma semana intensificada de treinamento, sugerindo a sua possível inclusão na rotina de atletas corredores amadores.

\section{REFERENCIAS}

1. Benvenutti, M. J., Alves E. D.S., Michael, S., Ding, D., Stamatakis, E., \& Edwards, K. M. (2017) A single session of hatha yoga improves stress reactivity and recovery after an acute psychological stress task-A counterbalanced, randomized-crossover trial in healthy individuals. Complementary Theraphy in Medice. 35:120-126.

2. Brandt, R., Bevilacqua, G. G., Andrade, A. (2007). Perceived sleep quality, mood states, and their relationship with performance among brazilian elite athletes during a competitive period. J Strength Cond Res. 31(4):1033-1039.

3. Brandt, R., Viana, M. S., Segato, L., \& Andrade, A. (2010) Estados de humor de velejadores durante o Pré-Panamericano. Motriz. 16(4): 834840 .

4. Brandt, R., Viana, S., Crocetta, T. B., Andrade, A. (2016). Association between mood states and performance of Brazilian elite sailors: Winners vs. non-winners. Cultura, Ciencia y Deporte. 11(32):119-125.

5. Calmeiro, L. Y., Tenenbaum, G. (2007) Fluctuations of cognitive-emotional states during competition: An idiographic approach. Revista de Psicología del Deporte. 16(1): 85-100.

6. Camelo, S. H. H., Angerami, E. L. S. (2004) Sintomas de estresse nos trabalhadores atuantes em cinco núcleos de saúde da família. Revista Latino-Americana Enfermagem. 12(1):14-21.

7. Cardoso, C. L., Loureiro, S. R., \& Nelson-Filho, P. (2004) Pediatric dental treatment: manifestations of stress in patients, mothers and dental school students. Brazilian Oral Research. 18(2):150-155.

8. Caulfield, M. J., Karageorghis, C. I. (2008) Psychological effects of rapid weight loss and attitudes towards eating among professional jockeys. Journal of Sports Sciences. 26(9): 877883.

9. Dallari, M. M. Corrida de rua: um fenômeno sociocultural contemporâneo. [Tese de 


\section{Rica RL, Maia AF, Leite GS, Miranda JMQ, Souza AS, Barbosa WA, Bocalini DS}

Doutorado]. São Paulo: Faculdade de Educação da Universidade de São Paulo; 2009.

10. Esfahani, N., Soflu, H, G,, Assadi, H. (2011). Comparison of mood in basketball players in Iran league 2 and relation with Team Cohesion and performance. In: Procedia - Social and Behavioral Sciences. 30: 2364-2368.

11. Ferreira Brandão, M. R., dos Santos Leite, G., Gomes, S. S., Figueira Júnior, A., Santo de Oliveira, R., \& Borin, J. P. (2015). Emotional alterations and the relation with training loads in high performance swimmers. Revista Brasileira de Ciências do Esporte. 37(4): 376-382.

12. Foster, C., Hector, L. L., Welsh, R., Schrager, M, Green, M. A., \& Snyder, A. C. (1995) Effects of specific versus cross-training on running performance. European Journal Applied Physiology. 70(4): 367-372.

13. Fry, R. W., Morton, A. R, \& Keast, D. (1992b) Periodisation and the prevention of overtraining. Canadian Journal Sports Science. 17(3): 241248.

14. Fry, R.W, Morton, A. R., \& Keast D. (1992a) Periodisation of training stress - a review. Canadian Journal Sports Science. 17(3): 234240.

15. Gomes, A. C. (2002) Treinamento desportivo: estrutura e periodização. São Paulo, Artmed.

16. Gomes, J. H., Mendes, R. R., Polito, L. F. T., Zanetti, M. C., Bocalini, D. S., Figueira Junior, A. J. (2018). Estado de humor e desempenho físico de jogadores jovens de basquetebol ao longo de uma competição. J. Phys. Educ. 29: e2969.

17. Gotaas, T. Correr: a história de uma das atividades físicas mais praticadas no mundo. São Paulo: Matriz; 2013.

18. Impellizzeri, F. M., Rampinini, E., Coutts, A. J., Sassi, A., \& Marcora, S. M. (2004) Use of RPE-based training load in soccer. Medicine and Science in Sports and Exercise. 36(6): 10421047.

19. Kentta, G., Hassmen, P., \& Raglin, J. S. (2006) Mood state monitoring of training and recovery in elite kayakers. European Journal of Sport Science. 6(4): 245-253.

20. Knechtli, B., Nikolaidis, P. T. (2018) Physiology and pathophysiology in ultramarathon duration. Frontiers of Physiology. 9: 634.

21. Leite, G. S., Sampaio, L. M. M., Serra, A. J., Miranda, M. L. J., Brandão, M. R. F., \& Wichi, R. B. (2012) Analysis of Knowledge Production about Overtraining Associated with Heart Rate Variability. JEPonline. 15(2): 20-29.

22. Lipp, M. E. N. (2005) Manual do Inventário de Sintomas de Stress para Adultos de Lipp (ISSL). São Paulo: Casa do Psicólogo.

23. Morgan, W. P., Brown, D. R., Raglin, J. S., O'Connor, P. J., \& Ellickson, K. A. (1987) Psychological monitoring of overtraining and staleness. British Journal of Sports Medicine, 25: 107-114.

24. Morgan, W. P., Costill, D. L., Flynn, M. G., Raglin, J. S., \& O'Connor, P. J. (1998) Mood disturbance following increased training in swimmers. Medicine Science in Sports and Exercise. 20: 408-414.

25. Oliveira, S. N. (2010) Lazer sério e envelhecimento: loucos por corrida. [Dissertação de Mestrado]. Porto Alegre: Universidade Federal do Rio Grande do Sul.

26. Rodrigues, D. F., Silva, A., Rosa, J. P. P., Ruiz, F. S., Verissímo, A. W., Winckler, C. A., Rocha, E. A., Parsons, A. A., Tufik, S., Mello, T. (2016). Profiles of mood states, depression, sleep quality, sleepiness, and anxiety of the Paralympic athletics team: A longitudinal study. Apunt Med l'Esport. 52(195):93-101.

27. Rohlfs, I.C.P.M., Carvalho, T., Rotta, T. M., \& Krebs, R. J. (2004) Aplicação de instrumentos de avaliação de estados de humor na detecção da síndrome do excesso de treinamento. Revista Brasileira de Medicina do Esporte. 10(2): 111116.

28. Rojo, J. R., Starepravo, F. A., Canan, F., Mezzadri, F. M., \& Silva, M. M. (2017) Transformações no modelo de corridas de rua no Brasil: um estudo na Prova Rústica Tiradentes. 


\section{Prática de Hatha Yóga e sintomas de estresse e humor de corredores}

Revista Brasileira de Ciência e Movimento. 25(1):19-28.

29. Salgado, J. V. V., Chacon-Mikahil, M. P. T. (2006) Corrida de rua: análise do crescimento do número de Provas e de praticantes. Revista Conexões. 4(1): 100-109.

30. Saw, A. E., Main, L. C., \& Gastin, P. B. (2016). Monitoring the athlete training response: subjective self-reported measures trump commonly used objective measures: a systematic review. British Journal of Sports Medicine. 50(5), 281-291.

31. Serra, A. J., Amaral, A. M., Rica, R. L., Barbieri, N. P., Reis Junior, D., Silva Junior, J. A., \& Bocalini D. S. (2009) Determinação da densidade corporal por equações generalizadas: facilidade e simplificação no método. ConScientiae Saúde. 8(1): 19-24.

32. Shipway, R., Holloway, I. (2016) Health and the running body: notes from an ethnography. International Review for the Sociology of Sports. 51(1): 78-96.

33. Shohani, M., Badfar, G., Nasirkandy, M. P., Kaikhavani, S., Rahmati, S., Modmeli, Y., \& Azami, M. (2018). The effect of yoga on stress, anxiety, and depression in women. International Journal of Preventive Medicine. 9: 21.

34. Suaay, F, Ricarte, J., Salvador, A. (1998) Indicadores psicológicos de sobretreinamento e agotamiento. Revista de Psicología del Deporte. 9(13): 7-28.

35. Terry, P. (1995). The Efficacy of Mood State Profiling with Elite Performers: A Review and Synthesis. Sport Psychol. 9(3): 309-324.

36. Terry, P. C., Lane, A.M. (2006).Use and perceived effectiveness of pre-competition mood regulation strategies among athletes. In: Proceedings of the 2006 Joint Conference of the Australian Psychological Society and the New Zealand Psychological Society. Psychology Bridging the Tasman: Science, Culture and Practice. 420- 424.

37. Tran, M. D., Holly, R. G., Lashbrook, B. S., Amsterdam, E. A. (2001) Effects of Hatha Yoga practice on the health- related aspects of physical fitness. Preventive Cardiology. 4(4):165-170.

38. Vieira, L. F., Oliveira, J. S., Gaion, P. A., Oliveira, H. G., Rocha, P. G. M., \& Vieira, J. L. L. (2010) Estado de humor e periodização de treinamento: um estudo com atletas fundistas de alto rendimento. Revista da Educação Física. 21(4): 585-591.

39. Wittig, A., McConell, G., Costill, D., \& Schurr, K. (1992). Psychological effects during reduced training volume and intensity in distance runners. International Journal of Sports Medicine. 13(06): 497-499. 